\title{
El arquitecto José Martínez Celiz y la introducción del barroco asturiano en Galicia
}

\author{
Javier Gómez Darriba \\ Universidade de Santiago de Compostela
}

\section{RESUMEN}

Durante la Edad Moderna no hubo una significativa interrelación entre la arquitectura del Principado de Asturias y la del Reino de Galicia pese a que eran territorios limítrofes. La excepción a esta regla la supuso José Martínez Celiz, un arquitecto de origen avilesino que introdujo en Galicia un repertorio de formas y modelos inéditos en la región, pero sobradamente trabajados en Asturias. Este personaje se asentó en la provincia de Mondoñedo en la primera mitad del siglo XVIII y allí llevó a cabo una prolífica actividad como tracista y aparejador. Puesto que su figura apenas resulta conocida por la historiografía, en el presente trabajo analizaremos su biografía, carrera profesional y personalidad artística.

\section{PALABRAS CLAVE}

José Martínez Celiz, arquitectura, Galicia, Asturias, siglo XVIII.

The architect José Martínez Celiz and the introduction of the Asturian Baroque in Galicia

ABSTRACT

During the Early modern period there wasn't significant interrelation between the architecture of the Principality of Asturias and that of the Kingdom of Galicia, despite the fact that they were bordering territories. The exception to this rule was José Martínez Celiz, an architect from Avilés who introduced into Galicia a repertoire of forms and models unpublished in the region, although amply worked in Asturias. He settled in the province of Mondoñedo in the first half of the $18^{\text {th }}$ century, and there he carried out a prolific activity as a draftsman and master builder. Since his figure is a little known in historiography, in this paper we will analyze his biography, professional career, and artistic personality.

José Martínez Celiz, architecture, Galicia, Asturias, $18^{\text {th }}$ century. 


\section{Introducción}

En la Galicia del siglo XVI y dos primeros tercios del XVII dejaron su huella grandes nombres de la arquitectura como Juan de Álava, Rodrigo Gil de Hontañón, Juan de Herrera (de Gajano), Mateo Lopes, Bartolomé Fernández Lechuga, José de la Peña de Toro, etc. Todos ellos provenían de reinos, regiones y comarcas foráneas como Portugal, Andalucía, Castilla, Vizcaya o la Merindad de Trasmiera. Su área de procedencia resultó fundamental, en tanto que introdujeron en el noroeste peninsular ciertas tipologías arquitectónicas, modelos estructurales u ornamentales propios de su lugar de origen, pero ajenos a Galicia. Sin embargo, en dicho periodo, el trasvase de artistas asturianos fue cuantitativamente residual, y se limitó a escasos ejemplos en la provincia de Mondoñedo, limítrofe con el Principado ${ }^{1}$. A partir del último tercio del siglo XVII y a lo largo del XVIII, el Reino de Galicia resultó la cuna de grandes arquitectos capaces de conformar un barroco regional ciertamente inconfundible. Por entonces la llegada de maestros provenientes de Asturias continuó siendo exigua. Apenas se circunscribió a la provincia mindoniense, procediendo todos ellos de Avilés. Entre los más destacados cabe mencionar a Francisco Menéndez Camina (hijo), quien entre 1708-1709 diseñó y ejecutó una canalización de aguas y una fuente para el claustro reglar del monasterio benedictino de Vilanova de Lourenzá2. A Manuel Álvarez

En la década de 1650 distintos canteros asturianos trabajaron a las órdenes de Antonio Rodríguez Maseda y Diego Ibáñez Pacheco en el primitivo convento de la Encarnación de Mondoñedo, GÓMEZ DARRIBA, Javier, " "Reconstruyendo" una ruina del siglo XVII. El desaparecido convento de la Encarnación de Mondoñedo", en Sémata, 31, Santiago de Compostela, 2019, pp. 257258. La historiografía ha mantenido en numerosas ocasiones que en 1637 cuatro maestros asturianos habian trabajado para Ibáñez Pacheco en las obras del claustro del monasterio benedictino de Vilanova de Lourenzá, pero la documentación asegura que dichos oficiales provenían del Valle del Liendo y que las trazas del mismo se debían a Juan de Villanueva, Archivo Histórico Provincial de Lugo (a partir de ahora A.H.P.L.), Protocolos Notariales, Bernabé García Cordido, leg. 8277-5, fols. 127r.-131v.

2 LENCE-SANTAR Y GUITIÁN, Eduardo, El Convento de Villanueva de Lorenzana y el de S. Francisco de Vivero, César G. Seco Romero, Mondoñedo, 1910, p. 24; CHAVARRÍA PACIO, César, El Monasterio de San Salvador de Lorenzana y su Museo de Arte Sacro, Celta, Lugo, 1984, p. 48; FERNÁNDEZ CASTIÑEIRAS, Enrique y FOLGAR DE LA CALLE, María del Carmen, “Magisteris,
Malleza, un maestro que trabajó en dicho cenobio y en el entorno de Mondoñedo entre 17291739³. A José Menéndez Camina, continuador del oficio que venía ejerciendo su línea paterna desde el siglo XVII, puesto que era nieto del citado Francisco, bisnieto del arquitecto homónimo e hijo de Bernardo Menéndez Camina, el cual también trabajó en Galicia en $1744^{4}$. A dicho José Menéndez, el obispo de Mondoñedo fray Antonio Alejandro Sarmiento le encargó en diciembre de 1749 el análisis pericial de las destartaladas casas que servían de Cárceles Eclesiástica y Seglar en la ciudad ${ }^{5}$. No sería

ordo e architectura. Las relaciones artísticas en el arco Atlántico a través del monasterio de San Salvador de Vilanova de Lourenzá”, en Galicia monástica. Estudos en lembranza da profesora María José Portela Silva, Universidade de Santiago de Compostela, Santiago de Compostela, 2009, pp. 595-596, 618-620; MADRID ÁLVAREZ, Vidal de la, Los Menéndez Camina y la arquitectura barroca en Asturias, Trea, Gijón, 2018, pp. 258-260.

3 En 1729 operó a las órdenes del arquitecto lego fray Lorenzo de Santa Teresa en el convento alcantarino de San Francisco del Rosal (Mondoñedo), GÓMEZ DARRIBA, Javier, "La arquitectura de la humildad. El convento alcantarino de San Francisco del Rosal en Mondoñedo", en Cuadernos de Estudios Gallegos, vol. 67, 133, Santiago de Compostela, 2020, p. 119. En enero de 1730 fue contratado por los benedictinos de Lourenzá para edificar junto con Sebastián Díaz Ribadeneira una celda tras la capilla mayor del templo, LENCE-SANTAR Y GUITIÁN, Eduardo, El Convento de Villanueva, opus cit., p. 27. En junio de 1734 tasó una casa en Mondoñedo, A.H.P.L., Protocolos Notariales, Mondoñedo, José Lozano y Cabo, leg. 5645-2, fols. 30r.-31v. En 1735, y de nuevo junto con Díaz Ribadeneira, pujó sin éxito por las obras de la sala capitular de la catedral mindoniense, GÓMEZ DARRIBA, Javier, La ciudad de Mondoñedo en los siglos XVII y XVIII. Construcción y nueva imagen de un centro de poder episcopal, Tesis Doctoral, Universidade de Santiago de Compostela, Santiago de Compostela, 2020, p. 182. Y entre diciembre de 1739 y enero del año siguiente continuó avecindado en Mondoñedo pese a que residía en su Avilés natal. Por aquel entonces había conseguido en concurso público la obra de la casa rectoral de la parroquia de San Cristóbal de Entreviñas (Zamora), y tenía más obras a su cargo, A.H.P.L., Protocolos Notariales, Ribadeo, Rosendo Martínez, leg. 1920-8, fols. 3r.-5v.

4 Se ignora en qué obra. El citado José compartía oficio con su hermano Juan, MADRID ÁLVAREZ, Vidal de la, La arquitectura de la Ilustración en Asturias. Manuel Reguera, 1731-1798, Real Instituto de Estudios Asturianos, Oviedo, 1995, pp. 187-188, 206-207; y del mismo autor Los Menéndez Camina, opus cit., pp. 82-83; SOLÍS SANTOS, Miguel, Sabugo. El barrio marinero de Avilés durante el Antiguo Régimen, Consejería de Cultura y Turismo, KRK, Oviedo, 2010, pp. 361-362.

5 LENCE-SANTAR Y GUITIÁN, Eduardo, Del Obispado de Mondoñedo, s.e., Mondoñedo, 1911, pp. 33-34; CAL PARD0, Enrique, Episcopologio Mindoniense, 
de extrañar que fuese asimismo el aparejador de la prisión que por aquel entonces se estaba construyendo en el arrabal de Los Remedios a expensas del prelado, pues parece inconcebible que acudiese desde Avilés para una cuestión tan baladí como el reconocimiento pericial de un par de inmuebles ${ }^{6}$. En definitiva, todos estos nombres ejercieron puntualmente su oficio en Galicia, pero ninguno lo hizo de una forma tan prolongada como José Martínez Celiz, quien, a diferencia de ellos, introdujo en sus obras rasgos propios de la arquitectura asturiana contemporánea.

\section{Biografía, carrera profesional y personalidad artística}

José Martínez Celiz se instaló en 1710 en la Mariña Lucense procedente de Coaña (Asturias). Debía de gozar de buena reputación en la cornisa cantábrica, pues en apenas un lustro tres órdenes religiosas de la provincia de Mondoñedo le confiaron el diseño y la ejecución de proyectos de suma relevancia. Paradójicamente no se sabe nada de su biografía antes de su llegada al Reino de Galicia. Ello denota el escaso interés que su figura ha despertado en la historiografía. Semejante desconocimiento llevó a algún autor a citarlo erróneamente como José Martínez Calvi ${ }^{7}$. Otros lo creyeron el artista que en el siglo XX pintó los muros de la iglesia de San Martiño de Vilalourente (Mondoñedo) ${ }^{8}$. Hubo que esperar a la década del 2000 para que apareciesen las primeras investigaciones rigurosas centradas en él. Las llevaron a cabo Fernández Castiñeiras y Folgar de la Calle, quienes perfilaron con buen criterio algunas de las características fundamentales de su personalidad

CSIC-Xunta de Galicia, Instituto de Estudios Gallegos "Padre Sarmiento", Santiago de Compostela, Estudios Mindonienses, Mondoñedo-Ferrol, 2003, pp. 751-752; GÓMEZ DARRIBA, Javier, La ciudad de Mondoñedo, opus cit., pp. 351-352, 362.

6 GÓMEZ DARRIBA, Javier, La ciudad de Mondoñedo, opus cit., pp. 368-369.

7 COUSELO BOUZAS, José, Galicia Artística en el siglo XVIII y primer tercio del XIX, Seminario, Compostela, 1932, p. 449.

8 GONZÁLEZ REBOREDO, José Manuel et al., Inventario artístico de Lugo y su provincia, Ministerio de Cultura, Madrid, 1980, t. 4, p. 193; GARCÍA IGLESIAS, José Manuel, Pinturas murais de Galicia, Xunta de Galicia, Santiago de Compostela, 1989, p. 52. artística $^{9}$. Respecto a su biografía, se ignora con certeza cuándo nació, aunque se supone que hacia 1680, puesto que en 1723 confesó tener 43 años ${ }^{10}$. Tampoco se conoce su lugar de origen, pero, como veremos, todo parece indicar que se formó en la comarca de Avilés. Aunque siempre firmaba como Celiz o Zeliz, dados sus apellidos, parece probable que se tratase de un hijo de Agustín Martínez Celis, o, en todo caso, de un pariente suyo ${ }^{11}$. En una reciente publicación, Vidal de la Madrid aportó algunos datos biográficos y artísticos acerca de este posible progenitor, al que la historiografía siempre ponderó como un maestro de obras vinculado a los Menéndez Camina. Falleció hacia 1698 siendo vecino de Avilés, pero era natural de la parroquia de Santa Eulalia de Ferroñes, en el concejo de Llanera. Sus padres habían sido Alonso Martínez y María Celis, y ambos procedían del mismo municipio asturiano. Agustín se desposó en 1679 con Manuela Álvarez del Carruébano, quien murió en $1688^{12}$. La carrera profesional de Agustín fue la de un maestro de obras que pujaba por la ejecución de diseños ajenos, especialmente de los Menéndez Camina. No sabía firmar y trabajó fundamentalmente en la comarca de Avilés, asumiendo la construcción de puentes, casas, capillas, fuentes o fachadas palaciegas como la de Camposagrado. También elaboró tasaciones de edificios ${ }^{13}$. Independientemente de la posible relación parental entre José y Agustín, cabe reseñar la existencia de otros maestros apellidados Celis en el noroeste peninsular en la segunda mitad del siglo XVII. Uno de ellos fue Francisco González Celis, un vecino de Labarces (Valdáliga, Cantabria) que

9 FOLGAR DE LA CALLE, María del Carmen, "La eclosión del Barroco", en Opus monasticorum. Patrimonio, arte, historia y orden, Xunta de Galicia, Santiago de Compostela, 2005, pp. 189-191; FERNÁNDEZ CASTIÑEIRAS, Enrique y FOLGAR DE LA CALLE, María del Carmen, "Magisteris, ordo", opus cit., pp. 596-609, 621-628.

10 GÓMEZ DARRIBA, Javier, La ciudad de Mondoñedo, opus cit., p. 471.

11 Ya se apuntó la posibilidad de que "pudiera tener algún vínculo familiar” con él en FERNÁNDEZ CASTIÑEIRAS, Enrique y FOLGAR DE LA CALLE, María del Carmen, "Magisteris, ordo", opus cit., p. 607.

12 MADRID ÁlVAREZ, Vidal de la, Los Menéndez Camina, opus cit., p. 167.

13 MADRID ÁlVAREZ, Vidal de la, "La arquitectura de Avilés en la Edad Moderna”, en Las ciudades españolas en la Edad Moderna: oligarquias urbanas y gobierno municipal, KRK, Oviedo, 2014, p. 473; y del mismo autor Los Menéndez Camina, opus cit., pp. 94-96, 167168, 195-198, 213. 
trabajó en la provincia de Lugo entre 1666 y $1671^{14}$. Ignoramos el parentesco que pudiera tener con el arquitecto que centra nuestra atención. Quizá ninguno. Lo que sí parece seguro a raíz de las últimas investigaciones es la nula vinculación de José Martínez Celiz con Juan de Celis, un maestro de obras muy activo en Asturias y que vivió entre $1605-1662^{15}$.

José Martínez Celiz llegó a la diócesis mindoniense a principios de 1710. Lo hizo con el objeto de pujar por una obra en el monasterio de San Salvador de Vilanova de Lourenzá. Concretamente la de un pabellón de tres alturas que cerrase el flanco norte del cenobio. La comunidad benedictina se la adjudicó. Dos años más tarde le encomendó el diseño y construcción de la cámara abacial y la portería monástica. Este edificio lo mantuvo ocupado entre 1713

14 BONET CORREA, Antonio, La arquitectura en Galicia durante el siglo XVII, CSIC, Madrid, 1966, p. 399; TAÍN GUZMÁN, Miguel, Domingo de Andrade. Maestro de obras de la catedral de Santiago (1639-1712), Ediciós do Castro, Sada (A Coruña), 1998, t. 1, p. 446; FERNÁNDEZ GASALLA, Leopoldo, La arquitectura en tiempos de Domingo de Andrade. Arquitectura y sociedad en Galicia (1660-1712), Tesis Doctoral, Universidade de Santiago de Compostela, Santiago de Compostela, 2004, pp. 622, 996-1002; CALLES LOMBA0, Marcos Gerardo, "La Torre de los Signos de la Santa Iglesia Catedral Basílica de Lugo (1575-1583), nuevos documentos localizados en los archivos lucenses", en Abrente, 49-50, A Coruña, 2017-2018, p. 30.

15 Acerca de su biografía y actividad artística véase RAMALLO ASENSIO, Germán, Escultura Barroca en Asturias, Instituto de Estudios Asturianos, Oviedo, 1985, pp. 182, 225; MADRID ÁLVAREZ, Vidal de la (coord.), El patrimonio artístico de Avilés, Casa Municipal de Cultura, Avilés, 1989, p. 160; KAWAMURA, Yayoi, Arquitectura y poderes civiles, Oviedo 1600-1680, Real Instituto de Estudios Asturianos, Oviedo, 2006, pp. 32, 52-54, 66, 102-104; y de la misma autora "Fábrica barroca del monasterio de Santa María de la Vega de Oviedo", en Liño, 13, Oviedo, 2007, pp. 38-40, 51-52; GARCÍA DEL BUSTO, Olmo, "La continuidad de las formas góticas en el siglo XVII asturiano. La reforma de la iglesia parroquial de San Nicolás de Bari en Avilés", en Liño, 16, Oviedo, 2010, pp. 21-32; GARCÍA DE TUÑóN AZA, Celso, "El arquitecto Juan de Celis y el palacio del marqués de Camposagrado en Mieres. Un capítulo esclarecido de la arquitectura barroca regional", en Anuario de la Sociedad Protectora de la Balesquida, 2, Oviedo, 2017, pp. 114-119; y del mismo autor "Juan de Celis (1605/1606-1662), arquitecto asturiano de la primera mitad del siglo XVII", en Anuario de la Sociedad Protectora de la Balesquida, 3, Oviedo, 2018, pp. 103-121; y también “Juan de Celis (1605/1606-1662), arquitecto asturiano de la primera mitad del siglo XVII: obras religiosas, públicas y diversas", en Anuario de la Sociedad Protectora de la Balesquida, 4, Oviedo, 2019, pp. 111-135. y 1715. Pero jamás lo concluyó tal y como lo había planteado porque los monjes se quedaron sin recursos (fig. 1). En el contrato se le exigió materializar la fachada con cantería proveniente de Gijón, Avilés u 0 Valadouro (Lugo). Dato sintomático de que estaba acostumbrado a trabajar en Asturias y a utilizar piedra de la zona $^{16}$. Pero lo que realmente denota su influjo asturiano es el diseño del frontispicio y la escalera interior, pues supone la traslación a Galicia de una serie de formas arquitectónicas, tanto estructurales como ornamentales, que se venían desarrollando en el Principado desde décadas atrás, gracias sobre todo a los arquitectos avilesinos Francisco Menéndez Camina (padre e hijo). De ahí que Fernández Castiñeiras y Folgar de la Calle hayan apuntado la posibilidad de que Martínez Celiz se hubiese formado con el primero, fallecido hacia $1694^{17}$. Ello no es del todo improbable, pues en aquella fecha era un adolescente de 13 años. Aun así pudo haberse instruido en el círculo del hijo homónimo, quien precisamente trabajó para el monasterio de Lourenzá entre 1708 y $1709^{18}$. Esto es, justo un año antes de que Celiz se estableciese en él para edificar el citado pabellón. No sería extraño que laborase aquí a las órdenes del avilesino

16 A.H.P.L., Protocolos Notariales, Mondoñedo, Diego Pumariño Lanzós, leg. 7162-1, fols. 136r.-140v.; LENCE-SANTAR Y GUITIÁN, Eduardo, El Convento de Villanueva, opus cit., p. 25; CHAVARRÍA PACIO, César, $E l$ Monasterio, opus cit., p. 43. Estas obras han sido ampliamente estudiadas por FERNÁNDEZ CASTIÑEIRAS, Enrique y FOLGAR DE LA CALLE, María del Carmen, “Magisteris, ordo", opus cit., pp. 596-607, 621-628.

17 FERNÁNDEZ CASTIÑEIRAS, Enrique y FOLGAR DE LA CALLE, María del Carmen, "Magisteris, ordo", p. 607. Aunque se ha escrito mucho sobre los Menéndez Camina, la publicación de referencia es MADRID ÁLVAREZ, Vidal de la, Los Menéndez Camina, opus cit. En ella se compila todo lo dicho hasta ahora sobre estos arquitectos y se ofrecen nuevas aportaciones. A dichos artífices también les ha dedicado un artículo monográfico BAS ORDÓÑEZ, Guillermo, "Los Menéndez Camina, Maestros de la Arquitectura Barroca Asturiana”, en Espacio, Tiempo y Forma. Serie VII, Historia del Arte, 24, Madrid, 2011, pp. 121-152. Pero en este trabajo no hay aportes significativos.

18 Véase la nota número 3. Cabe la posibilidad de que el arquitecto leonés fray Gabriel de Casas acudiese también a Lourenzá a pujar por la adjudicación de esta obra o si acaso a valorar las trazas y condiciones de Menéndez Camina, pues el avilesino firmó la escritura notarial correspondiente a sus obligaciones el 12 de marzo, y el 24 del mismo mes el lego se presentó en Mondoñedo a petición del obispo fray Juan Muñoz y Salcedo después de que este se hubiese enterado de que se hallaba en Lourenzá, GÓMEZ DARRIBA, Javier, “"Reconstruyendo» una ruina”, opus cit., pp. 268-269. 


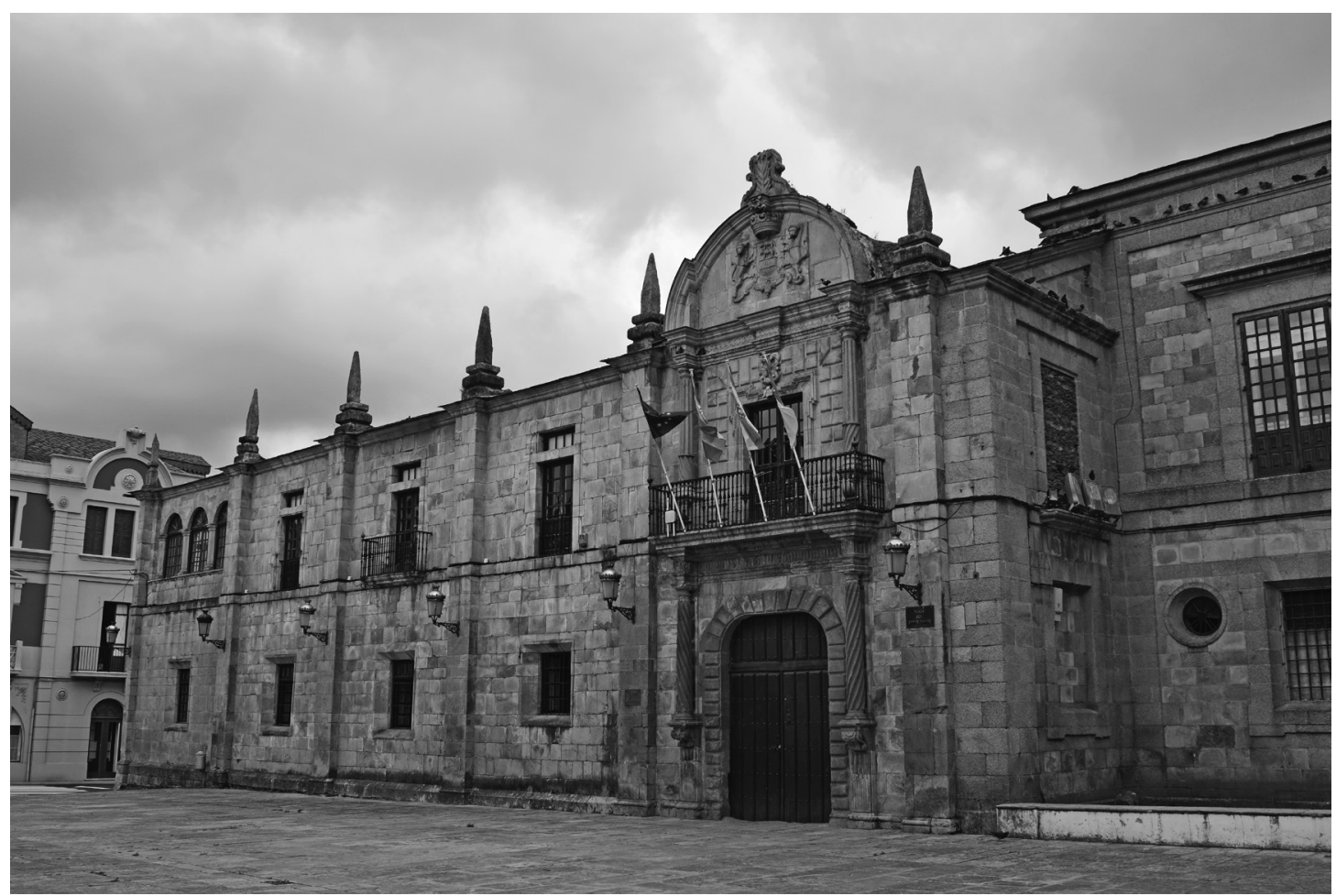

Fig. 1. José Martínez Celiz, fachada de la portería y de la cámara abacial del monasterio de San Salvador de Vilanova de Lourenzá, 1712-1715, fotografía del autor.

y que esta fuese su tarjeta de presentación para regresar en 1710. Sea como fuere, el diseño de la fachada evidencia un profundo conocimiento de la arquitectura asturiana de la época. Compárese si no la calle donde se abre su portada con la central de la fachada meridional del Palacio de Camposagrado en Avilés ${ }^{19}$. Este frontispicio había sido contratado en 1693 a los maestros Agustín Martínez Celis, Domingo de Festas e Isidoro Martínez. La historiografía no atribuye su diseño a ninguno de ellos por su discreto currículum. Cree más bien que hubo de deberse a Francisco Menéndez Camina (padre), no solo por cuestiones formales, sino porque ya había trabajado para dicha casa años antes ${ }^{20}$.

19 Esto ya se puso de manifiesto en FOLGAR DE LA CALLE, María del Carmen, "La eclosión del Barroco", opus cit., pp. 189-190; FERNÁNDEZ CASTIÑEIRAS, Enrique y FOLGAR DE LA CALLE, María del Carmen, "Magisteris, ordo", opus cit., p. 607.

20 RAMALLO ASENSIO, Germán, Arquitectura civil asturiana (Época moderna), Ayalga, Gijón, 1978, pp. 133-140; y del mismo autor "Las casas urbanas de la familia Camposagrado", en II Simposio sobre el Padre Feijoo y su siglo. (Ponencias y comunicaciones), Centro de Estudios del S. XVIII, Oviedo, 1983, t. 2, pp. 576579; y también "El palacio urbano en Asturias", en Arquitectura señorial en el norte de España, Universidad
La superposición de alturas articuladas con columnas, la utilización de fustes melcochados, el juego de puertas ventanas abiertas a un balcón, el perímetro de los vanos revestido con almohadillado o el modo de disponer el blasón en la peineta son ciertamente similares. Los leones que flanquean el escudo resultan prácticamente idénticos a los presentes en la fachada del palacio avilesino del Marqués de Ferrera, diseñada a mediados del XVII por Bartolomé de Velasco $^{21}$. Algunas de las características mencionadas también retrotraen a la calle principal de la fachada del monasterio asturiano de San Salvador de Cornellana, en Salas ${ }^{22}$, obra adjudicada en 1694 a los maestros avilesinos Fran-

de Oviedo, Oviedo, 1993, p. 96; MADRID ÁLVAREZ, Vidal de la, "El palacio del marqués de Camposagrado en Avilés (Asturias) y la muralla de la villa a finales del siglo XVII", en Liño, 16, Oviedo, 2010, pp. 67-83; y del mismo autor Los Menéndez Camina, opus cit., pp. 156178.

${ }_{21}$ Sobre este inmueble véase MADRID ÁLVAREZ, Vidal de la, El Palacio del Marqués de Ferrera, Trea, Gijón, 2003.

22 FOLGAR DE LA CALLE, María del Carmen, "La eclosión del Barroco", opus cit., p. 190; FERNÁNDEZ CASTIÑEIRAS, Enrique y FOLGAR DE LA CALLE, María del Carmen, "Magisteris, ordo", opus cit., p. 607. 
cisco González Bango y Domingo Suárez Solar, pero cuyo diseño se considera de Francisco Menéndez Camina (hijo) ${ }^{23}$. Igualmente, la escalera interior de la cámara de Lourenzá se ha vinculado tipológicamente con otras presentes en el Principado, caso de la del primer proyecto del palacio ovetense de Camposagrado ${ }^{24}$, diseñada hacia 1698 y considerada también del mismo Menéndez Camina ${ }^{25}$. La escritura contractual del edificio de Vilanova obligaba a Celiz a trabajar allí desde la primavera de 1713 hasta septiembre de 1715. Según su propia declaración estas obras lo mantuvieron ocupado durante 23 meses, de ahí que se avecindase en la villa ${ }^{26}$. Posiblemente a inicios de 1715 estas entraron en un declive que derivó en el incumplimiento del proyecto original, pues en febrero los franciscanos terceros de Mondoñedo lo contrataron por un plazo de catorce meses para que edificase la iglesia de San Martiño de Vilalourente que él mismo había delineado ${ }^{27}$.

Con anterioridad, hacia el primer semestre de 1713 , las monjas concepcionistas de Mondoñedo le habían encargado el diseño del nuevo convento de la Encarnación. Esta obra se inició entonces y se culminó en $1717^{28}$. En ella no trabajó como aparejador, pues estaba comprometido con los benedictinos de Lourenzá

23 MADRID ÁLVAREZ, Vidal de la, Los Menéndez Camina, opus cit., pp. 212-222. La utilización del fuste melcochado resultó inhabitual en la arquitectura pétrea del barroco gallego. Sin embargo, sí tuvo cabida en las obras asturianas citadas. En el Principado ya se aprecia su uso a mediados del siglo XVI en la portada de la iglesia de San Emeterio de Sietes (Villaviciosa), GARCÍA CUETOS, María Pilar, Arquitectura en Asturias, 1500-1580. La dinastía de los Cerecedo, Real Instituto de Estudios Asturianos, Oviedo, 1996, pp. 58-61.

24 FERNÁNDEZ CASTIÑEIRAS, Enrique y FOLGAR DE LA CALLE, María del Carmen, "Magisteris, ordo", opus cit., pp. 603-609.

25 MADRID ÁLVAREZ, Vidal de la, Pedro Antonio Menéndez. Un arquitecto entre el Barroco y la Ilustración, Azucel, Avilés, 1997, pp. 35-39; y del mismo autor Los Menéndez Camina, opus cit., pp. 222-226. Acerca de la decoración empleada por los Menéndez Camina véase RAMALLO ASENSIO, Germán, "El decorativismo en la arquitectura barroca asturiana. Los Menéndez Camina”, en I Semana del Patrimonio Artístico Asturiano. Oviedo del 20/25 noviembre 1978. Ponencias e intervenciones, Caja de Ahorros de Asturias, Oviedo, 1979, pp. 83-96.

26 Véase la nota número 17.

27 GÓMEZ DARRIBA, Javier, "El convento de San Martiño de Vilalourente en Mondoñedo. La transformación moderna de un cenobio medieval", en BSAA Arte, 86, Valladolid, 2020, pp. 266-267.

28 GÓMEZ DARRIBA, Javier, La ciudad de Mondoñedo, opus cit., pp. 260-264. en construir la cámara abacial; y desde 1715 con los frailes terceros en erigir la citada iglesia de San Martiño, la cual levantó siguiendo una "ydea y planta" suyas ${ }^{29}$. En los dos cenobios mindonienses se eludió la riqueza decorativa y la apariencia palaciega de la fachada y escaleras de Vilanova. La tosquedad que exhiben ambas fábricas tampoco guarda relación con la mayor calidad estereotómica que luce el edificio benedictino. Esto se explica por las dificultades financieras que tuvieron las concepcionistas y los terciarios a la hora de construir sus respectivas casas monásticas, cuya conclusión solo fue posible gracias al patrocinio del obispo fray Juan Muñoz y Salcedo. En el diseño de ambos conventos Martínez Celiz incluyó notas características de la arquitectura asturiana de los siglos XVII y XVIII. Por ejemplo, en el entablamento de la portada de la iglesia de la Encarnación empleó un friso con triglifos y metopas en las cuales inscribió unas rosetas (fig. 2). De esta manera, siguió un planteamiento similar al de la portada principal del templo jesuita de San Matías en Oviedo, obra en la que intervino Francisco Menéndez Camina (padre) en el último tercio del seiscientos ${ }^{30}$. También recuerda al friso del segundo cuerpo de la citada portada del monasterio de Cornellana. 0 a las impostas de la fachada meridional del aludido palacio avilesino de Camposagrado. En la portada de la iglesia de San Martiño recurrió al uso de la sillería almohadillada para revestir el cajeado de las pilastras (fig. 3). Estas retrotraen a las del Claustro de las Oficinas del monasterio compostelano de San Martín Pinario, edificio que seguramente no conoción ${ }^{31}$. Lo cierto es que los arquitectos asturianos del momento, entre los cuales se incluye Celiz, emplearon con frecuencia el almohadillado para guarnecer los enmarques de puertas, ventanas, esquinales,

\footnotetext{
29 En la escritura notarial apareció como testigo un vecino de la parroquia de Santa María de Berbes (Ribadesella), A.H.P.L., Protocolos Notariales, Mondoñedo, Antonio Fernández de Parga, leg. 6856-3, fols. 10r.-11r., 173r. Este documento lo dio a conocer LENCE-SANTAR Y GUITIÁN, Eduardo, Mondoñedo. El Convento de San Martín de Villaoriente o de los Picos, Sucr. de A. Villamarín, Lugo, 1912, p. 18.

30 MADRID ÁLVAREZ, Vidal de la, Los Menéndez Camina, opus cit., pp. 98-112.

31 Acerca del debate sobre la autoría de dicho claustro véase VICENTE LÓPEZ, Simón, Vega y Verdugo, Peña de Toro y la introducción del barroco en Compostela, Consorcio de Santiago, Teófilo, Santiago de Compostela, 2012, pp. 68-70.
} 


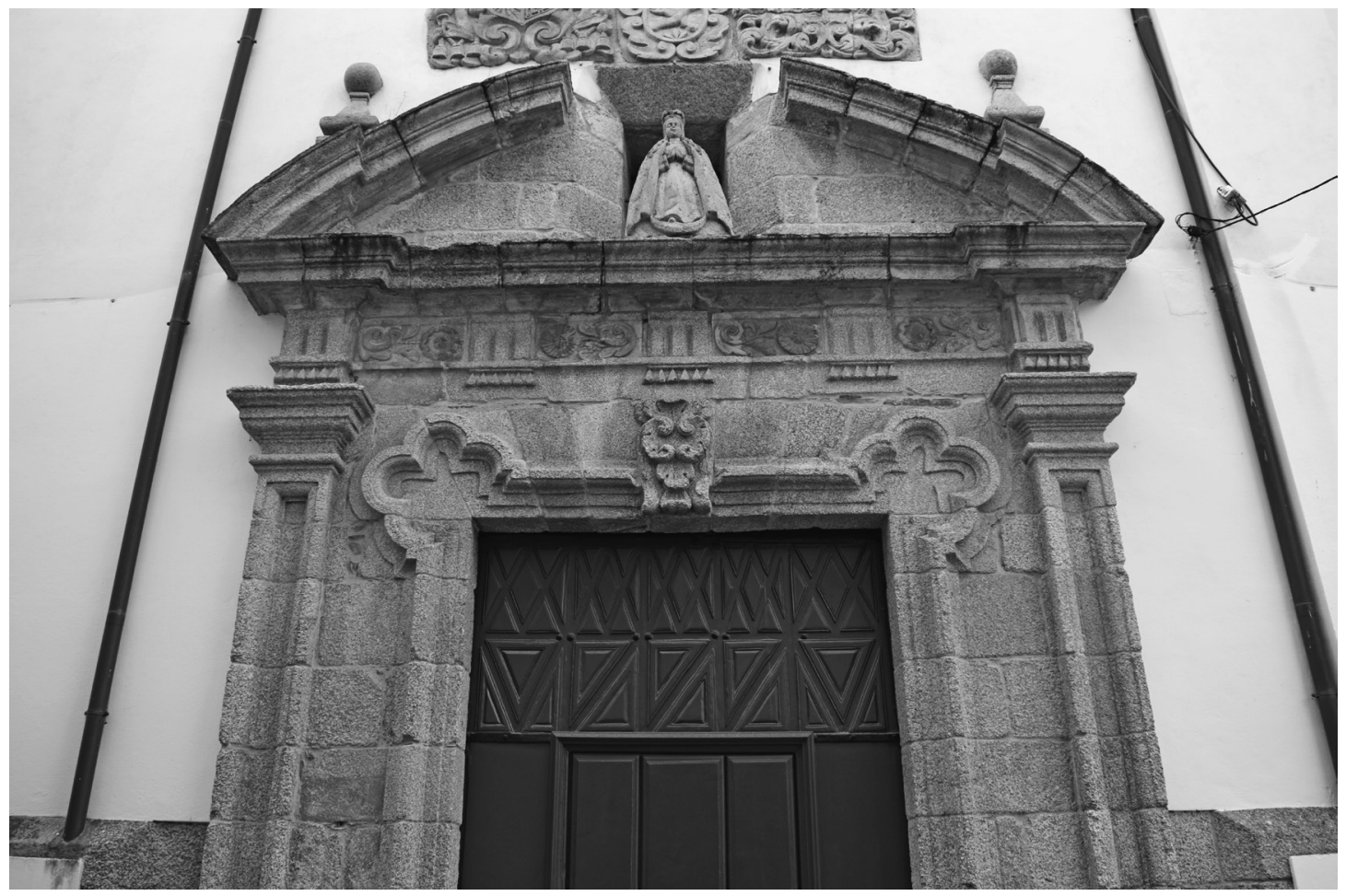

Fig. 2. José Martínez Celiz, portada de la iglesia conventual de la Encarnación en Mondoñedo, 1713-1717, fotografía del autor.

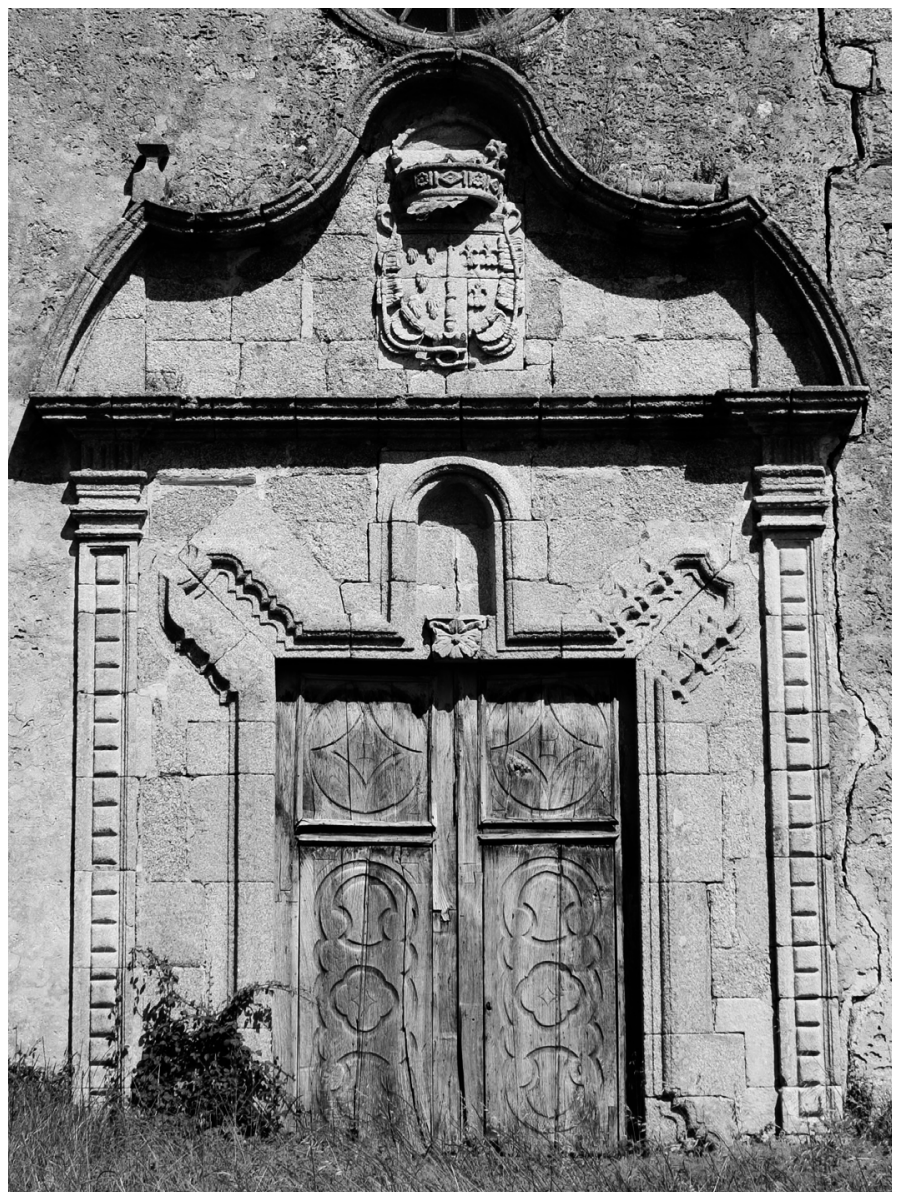

Fig. 3. José Martínez Celiz, portada de la iglesia conventual de San Martiño de Vilalourente en Mondoñedo, 1715-1716, fotografía del autor. 
etc. Pero, sin lugar a dudas, la nota más característicamente asturiana de las dos portadas la exhiben sus marcos moldurados, fundamentalmente en lo tocante al quiebro mixtilíneo que dibujan todos sus ángulos, inclusive los que alcanzan el suelo. El desarrollo de los de la fachada del convento de San Martiño es ciertamente extraordinario. Esta manera tan peculiar y anticlásica de conjugar las orejeras bebe en última instancia de los vanos del primer piso de la fachada de la vicaría del monasterio de San Pelayo en Oviedo, diseñada en 1703 por fray Pedro Martínez de Cardeña y levantada por los maestros Alonso Pérez y Toribio Díaz ${ }^{32}$. A partir de aquí se repite en distintos palacios asturianos, caso del ovetense del Duque del Parque o del gijonés de Revillagigedo ${ }^{33}$. La historiografía considera que este último se construyó entre 1705-1723 siguiendo un diseño de Francisco Menéndez Camina (hijo) ${ }^{34}$. Mientras que el de Oviedo es levemente posterior a los conventos de Mondoñedo, pues lo proyectó Francisco de la Riva hacia 1723 y no se concluyó hasta $1730^{35}$. Aquí las ventanas del piso superior de la fachada principal presentan unos quiebros en las orejeras más tenues que los mindonienses. No muy lejos de Mondoñedo, a unos $22 \mathrm{~km}$, se halla en la parroquia de Santiago de Abres (Vegadeo) el Palacio de El Pividal, en cuya fachada la portada principal y la ventana superior reiteran esta tipología de enmarque, si bien no se asemeja tanto al característico de Martínez Celiz como sí al del Palacio de Revillagigedo.

32 RAMALLO ASENSIO, Germán, “Documentación y estudio de la obra realizada por Fray Pedro Martínez de Cardeña en el monasterio de San Pelayo de Oviedo”, en Boletín del Instituto de Estudios Asturianos, 87, Oviedo, 1976, pp. 198-204.

33 SCHUBERT, Otto, Historia del Barroco en España, Saturnino Calleja, Madrid, 1924, pp. 282-283; RAMALLO ASENSIO, Germán, "Documentación y estudio", opus cit., p. 193; y del mismo autor Arquitectura civil, opus cit., pp. 144-149.

34 RAMALLO ASENSIO, Germán, Arquitectura civil, opus cit., pp. 149-154; y del mismo autor "El palacio urbano", opus cit., pp. 102, 104-105; MADRID ÁLVAREZ, Vidal de la, Palacio Revillagigedo y Colegiata de San Juan Bautista, Caja de Ahorros de Asturias, Gijón, 1991-1992, pp. 13-15, 27; y del mismo autor "Arquitectura barroca civil (II)", en El arte en Asturias a través de sus obras, Prensa Asturiana, Oviedo, 1996, pp. 219-221; y también Los Menéndez Camina, opus cit., pp. 235-252.

35 RAMALLO ASENSIO, Germán, Arquitectura civil, opus cit., pp. 148, 162-167; y del mismo autor "El palacio urbano”, opus cit., pp. 101-102; MADRID ÁLVAREZ, Vidal de la, El arquitecto barroco Francisco de la Riva Ladrón de Guevara (1686-1741), Trea, Gijón, 1998, pp. 59-81.
Otro ejemplo posterior que muestra estos ángulos mixtilíneos, tanto en las orejeras como en los pies de la portada, se halla en la Lonja de la catedral de León, realizada en 1750 y atribuida al trasmerano Fernando de Compostizo ${ }^{36}$. También cabe destacar, por último, el paralelismo entre los quiebros de la portada de la iglesia de Vilalourente y la decoración de las yeserías que revisten el interior del antiguo templo parroquial de San Julián de Astorga, obra de autor y fecha desconocidos, aunque considerada de la primera mitad del siglo XVIII ${ }^{37}$.

En el transepto de los templos conventuales de Mondoñedo, Celiz abrió una media naranja asentada en pechinas, tal y como había hecho en el interior de la cámara abacial de Lourenzá (fig. 4). El modelo de cúpula sobre pechinas culminada por linterna octogonal -utilizado en Mondoñedo- se repite en la capilla del Cristo de la iglesia avilesina de los Padres Franciscanos, pero dicho recinto es levemente posterior, pues se erigió entre 1728-1729 y se desconoce su autoría ${ }^{38}$. Celiz articuló los muros interiores de dichos templos con pilastras toscanas, cuyo fuste cajeado no resulta común en la arquitectura gallega, pues lo rehúnde una fina ranura. Una solución similar se aprecia en algunas de las pilastras del interior de la catedral de Valladolid, o, volviendo a Avilés, en la fachada de la antigua casa de García Pumarino, construida entre 1700-1706 por Francisco Menéndez Camina (hijo) y Domingo Suárez Solar, aunque su diseño se atribuye al primero ${ }^{39}$. Respecto a la cubrición de los conventos mindonienses, Celiz apostó en el de la Encarnación por bóvedas de aristas para los tramos de la nave, y de cañón para la escueta capilla mayor. Sin embargo, en el templo de Vilalourente recurrió a una bóveda de lunetos cuya singularidad estriba en la presencia de nervios con medallones en sus claves, que en última instancia remiten a la arquitectura hispana de tradición gótica tan habitual en

\footnotetext{
36 MORAIS VALLEJO, Emilio y CAMPOS SÁNCHEZ-BORDONA, María Dolores, Arquitectura y patrimonio. Edificios civiles de la ciudad de León en la Edad Moderna, Universidad de León, León, 2007, pp. 100-109.

37 MORAIS VALLEJO, Emilio, Aportación al Barroco en la provincia de León. Arquitectura religiosa, Universidad de León, León, 2000, pp. 371-375.

38 MADRID ÁLVAREZ, Vidal de la (coord.), El patrimonio artístico, opus cit., pp. 163-165.

39 MADRID ÁLVAREZ, Vidal de la (coord.), El patrimonio artístico, opus cit., p. 245; y del mismo autor "Arquitectura barroca”, opus cit., pp. 217-219; y también Los Menéndez Camina, opus cit., pp. 226-235.
} 


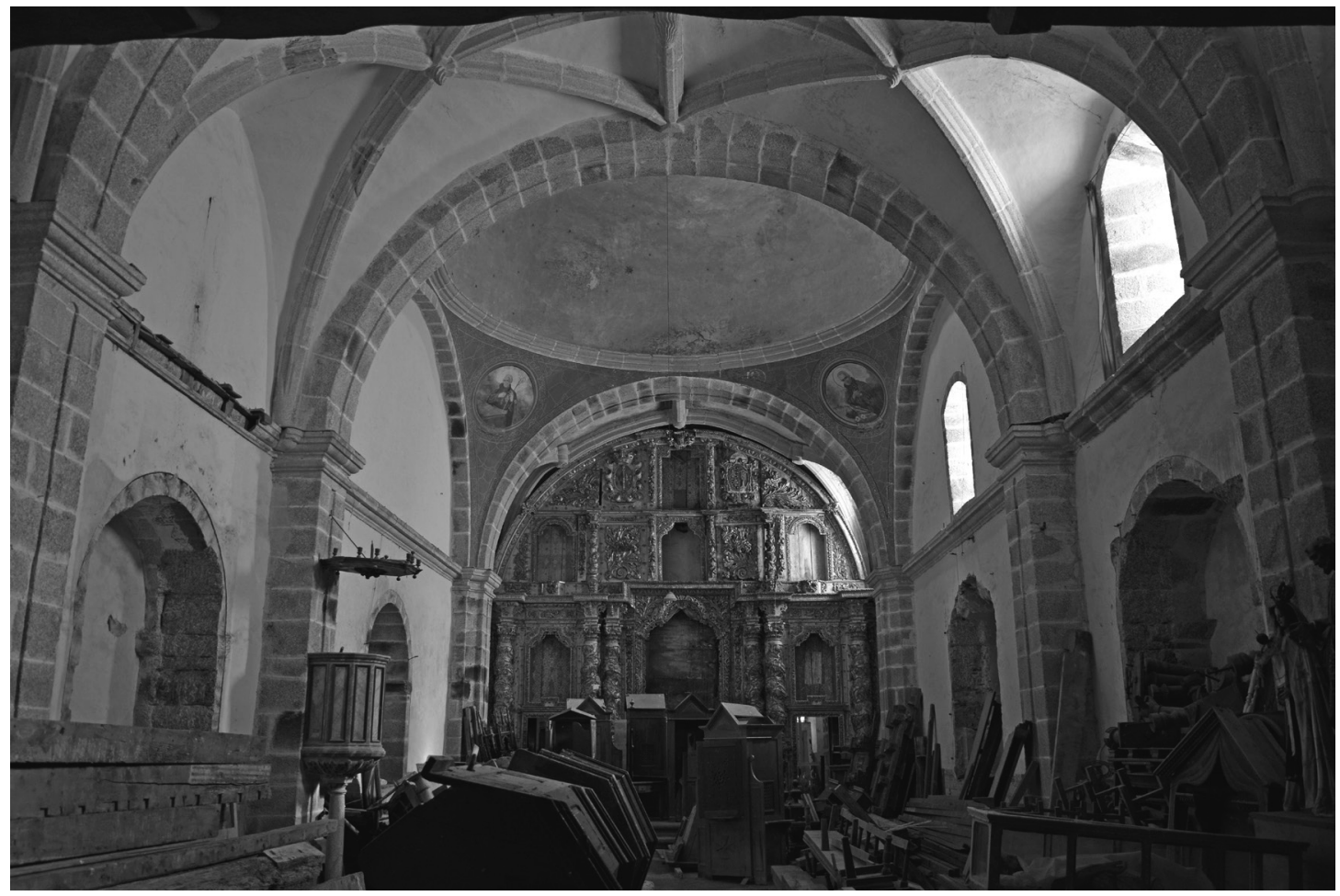

Fig. 4. José Martínez Celiz, interior de la iglesia conventual de San Martiño de Vilalourente en Mondoñedo, 1715-1716, fotografía del autor.

el siglo XVI. Lo cierto es que en muchas iglesias dieciochescas de la cornisa cantábrica pervivió una técnica de abovedamiento propia del quinientos ${ }^{40}$. Lo más sorprendente de esta cuestión resulta la comparación entre este templo y el de la parroquia de Santa Marina de Puerto de Vega, en Navia, pues son muy parejos en lo que respecta a su interior. La nave, el crucero y la capilla mayor de este último se edificaron entre 1730-1737 (fig. 5) ${ }^{41}$. Desgraciadamente se desconoce quién diseñó y ejecutó esta parte. Solo consta que los maestros avilesinos José Menéndez Camina y José Muñiz concluyeron la fachada en los últimos años de la década de $1740^{42}$. No sería de extrañar, por tanto, que el interior se debiese a un proyecto de José Martínez Celiz, pues pese a que la parroquia tiene brazos en su crucero, por lo demás casi resulta un calco del templo mindoniense. Ambas iglesias cuentan con un tipo de bóveda de lunetos

40 GÓMEZ MARTÍNEZ, Javier, El gótico español de la Edad Moderna. Bóvedas de crucería, Universidad de Valladolid, Valladolid, 1998, pp. 227-231.

41 PENZOL, Pedro, "Descripción de la Iglesia de Santa Marina de Puerto de Vega", en Boletín del Instituto de Estudios Asturianos, 11, Oviedo, 1950, pp. 213-214.

42 MADRID ÁlVAREZ, Vidal de la, Los Menéndez Camina, opus cit., p. 82. muy similar, con nervios y medallones en las claves. También con idénticos arcos fajones apenas rehundidos por una sucinta ranura. Otra cuestión que se reitera es la articulación mural, pues se usan pilastras toscanas con la misma hendidura en su fuste. Estas se achaflanan en el crucero, y sobre ellas se apoyan las pechinas en las que monta la media naranja, que en el caso asturiano carece de claraboya.

Conocida la procedencia de Martínez Celiz, las principales obras que llevó a cabo en la antigua provincia de Mondoñedo, o sus rasgos estilísticos más singulares, cabe mencionar otros aspectos de su vida y profesión a fin de redondear su perfil como arquitecto y aparejador. Como se dijo, aunque entre 1710-1712 declaró ser vecino de Coaña, en esos años fijó su residencia en Vilanova de Lourenzá, y de esta no se movió durante casi dos décadas pese a sus estancias laborales en Mondoñedo. De hecho en 1722 era merino de aquella localidad ${ }^{43}$. Hallándose en ella, fue reclamado en 1714 por el Cabildo mindoniense junto con el escultor Bernardo de Amieiro para esclarecer si convenía o

\footnotetext{
${ }_{43}$ En septiembre se le cita como "merino justt. ${ }^{a}$ hordin. ." $^{\text {", }}$ A.H.P.L., Protocolos Notariales, Mondoñedo, Juan Antonio de Villar y Rubiños, leg. 7383-4, fol. 282r.
} 


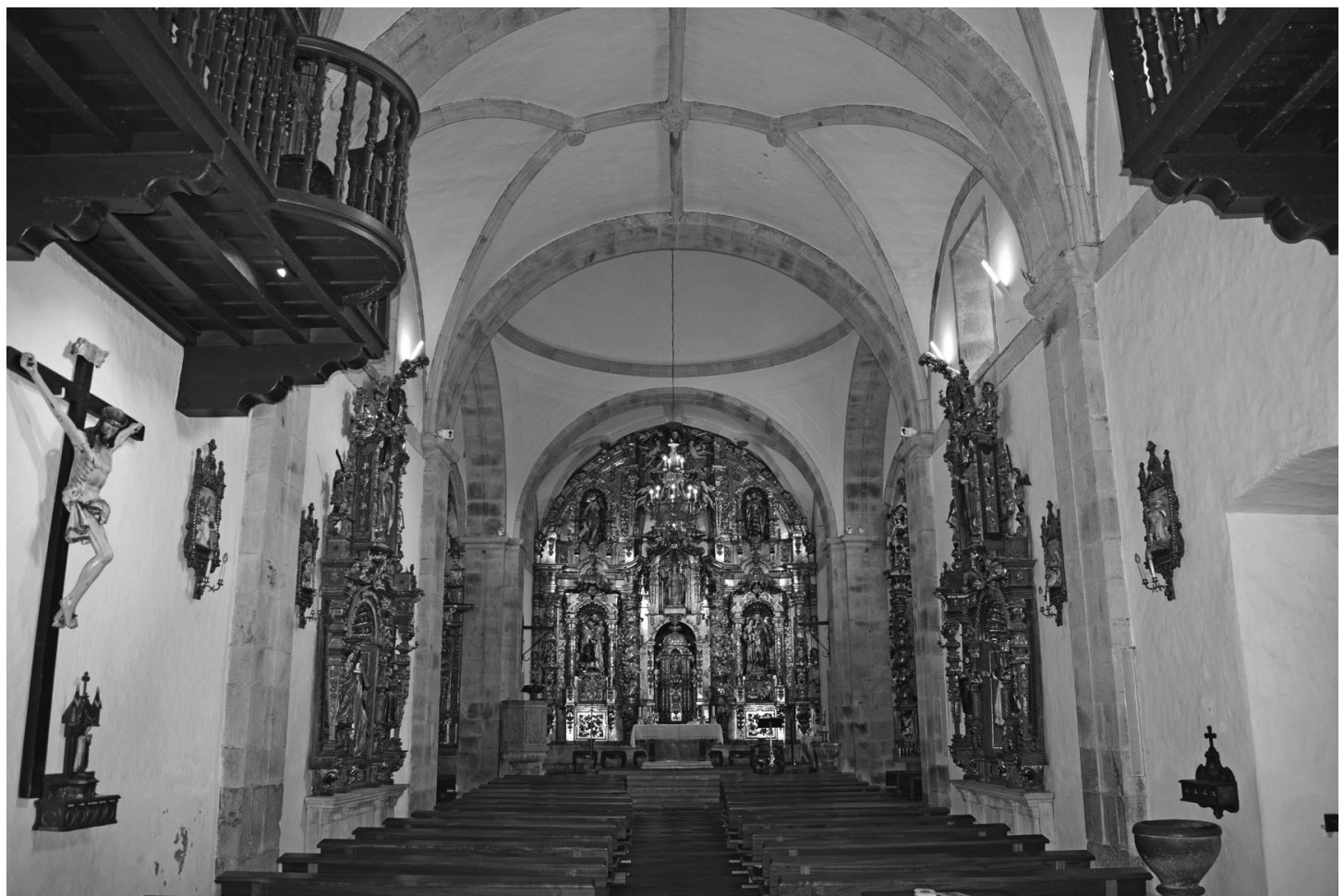

Fig. 5. ¿José Martínez Celiz?, interior de la iglesia parroquial de Santa Marina de Puerto de Vega en Navia, 1730-1737, fotografía del autor.

no alargar el coro catedralicio a fin de disponer sobre el mismo unos nuevos órganos ${ }^{44}$. Al año siguiente efectuó la tasación de un palacete urbano en la capital episcopal junto con su colega Bartolomé Fernández, vecino "del conzexo de santiso" (San Tirso de Abres, Asturias), y el carpintero Lorenzo García Cabodevila, de Ribadeo ${ }^{45}$. En mayo de 1716 se concertó con Pedro Pardo de Andrade, dueño de la Casa de Baltar, para reformar su vivienda. Tendría que demoler buena parte de la antigua y conservar la planta baja de la fachada, hecha en piedra. Sobre esta alzaría dos pisos en ladrillo y colocaría en el segundo un balcón de madera con sus balaustres $^{46}$. En 1718 acudió a Asturias para proyectar

44 Archivo de la Catedral de Mondoñedo, Actas Capitulares, vol. 15, fols. 540r.-541r.; CAL PARD0, Enrique, La música de la Catedral de Mondoñedo, Alvarellos, Lugo, 1996, p. 287; y del mismo autor La Catedral de Mondoñedo. Historia, s.e., Lugo, 2002, p. 38; y también Episcopologio Mindoniense, opus cit., pp. 672-673.

45 Esta casona tenía dos fachadas. Una miraba hacia la Rúa del Perejil -actual Pardo de Cela- y otra a la del Colegio -hoy Alfonso VII-. Estimaron su arreglo en 16.300 reales, Arquivo do Reino de Galicia, Real Audiencia, leg. 7818-12, fols. 311v., 322r., 325v., 327v., 329v.-334r.

46 También haría una cocina, salones y alcobas. La obra tendría que estar acabada para el inmediato mes de la reedificación de un puente sobre el río Porcía, en El Franco. Hizo la traza y condiciones de la obra y la presupuestó en casi 23.000 reales $^{47}$. En 1723 el Ayuntamiento de Mondoñedo y el obispo fray Juan Muñoz lo requirieron junto con el maestro Sebastián Díaz Ribadeneira para que diseñase dos obras de suma relevancia en la ciudad. Por un lado una canalización de aguas y una serie de fuentes; y por otro una flamante Casa Consistorial. Respecto a la conducción de aguas el prelado nunca terminó de fiarse de la propuesta de Celiz. De hecho requirió del arquitecto benedictino fray Francisco Velasco para que la examinase, y finalmente encomendó el diseño de una nueva canalización al lego cisterciense fray Agustín de Otero ${ }^{48}$. El edificio del Consistorio, por su parte, no pasó del mero pro-

octubre. El promotor le daría buena parte de los materiales y 4.500 reales de vellón. Firmaron por Martínez Celiz -que entonces continuaba avecindado en Lourenzá- José de Sande y Manuel Roel, vecinos de Mondoñedo, A.H.P.L., Protocolos Notariales, Mondoñedo, Antonio Fernández de Parga, leg. 6856-4, fols. 98r.-99v.

${ }_{47}$ CADIÑANOS BARDECI, Inocencio, "Noticias sobre algunos puentes asturianos en la Edad Moderna”, en Liño, 14, Oviedo, 2008, pp. 57, 62.

${ }^{48}$ GÓMEZ DARRIBA, Javier y PITA GALÁN, Paula, "Fray Agustín de Otero. Monje cisterciense y arqui- 
yecto. Por desgracia no se conservan las trazas, aunque se sabe que Celiz ideó un inmueble con arquerías en sus dos fachadas, balcones volados con balaustres de hierro y canalones con gárgolas en las esquinas. En este sentido, parece como si quisiera erigir una Casa Consistorial similar a la original, construida entre 15691584 por el maestro vizcaíno Pedro de Artiaga, la cual llegó a 1723 con distintas alteraciones. En su interior pretendía abovedar por entero el piso principal, hacer las paredes de mampostería y el suelo de ladrillo. La obra se presupuestó en 6.000 ducados, pero, como se dijo, jamás se llegó a efectuar ${ }^{49}$. La última noticia segura que concierne a su figura data de entre 1729-1732. Entonces acudió a Mondoñedo el "maestro de obras de Villanueba Martínez [...] a reconocer la ruina q amenazaba" la ermita de Nuestra Señora de los Remedios "cuando el P. ${ }^{\text {e }}$ fr Lorenzo de s. $^{\text {ta }}$ theresa aseguro estar de peligro" ${ }^{\circ 0}$. De este escueto dato se desprende lo siguiente: el obispo Sarmiento estaba volcado en aquellos años en el avance de las obras del convento alcantarino de San Francisco del Rosal, fundado por su predecesor en la cátedra -fray Juan Muñoz-, y recurrió al maestro que las dirigía -fray Lorenzo de Santa Teresa-, para que analizase el estado del templo mariano habida cuenta de que él, como obispo, era patrono del mismo. Puesto que la valoración del franciscano no resultó positiva, el prelado quiso cerciorarse y para ello recurrió a un arquitecto experimentado como lo era Martínez Celiz. Es posible que este maestro o el propio fraile fuesen los encargados de proyectar la nueva iglesia, cuyas obras se iniciaron en junio de 1733 y se prolongaron hasta junio de 1738, tal y como indican las cartelas de su fachada. Pero conocida la personalidad artística del arquitecto asturiano, no hay nada en el templo que muestre similitud con ninguna de sus obras ${ }^{51}$. Desde entonces

tecto en la Galicia del Barroco", en Atrio, 26, Sevilla, 2020, pp. 137-141.

49 NOVO SÁNCHEZ, Francisco Javier, "Antigua casa consistorial (Mondoñedo)", en Arquitecturas desvanecidas. Memoria gráfica del patrimonio desaparecido en Galicia, Abada, Madrid, 2019, pp. 332-334; GÓMEZ DARRIBA, Javier, "Los Artiaga: la actividad artística de unos maestros canteros vizcaínos en Galicia (15601600)", en Ars Bilduma, 11, Vitoria, 2021, pp. 111-117.

50 Por esta visita pericial cobró 15 reales, Archivo Histórico Diocesano de Mondoñedo-Ferrol, Remedios, Fábrica, n. ${ }^{\circ}$, cuentas $1729-1732$, s.f.

51 Recientemente se ha relacionado el diseño de esta iglesia con el lego benedictino fray Juan Vázquez. Sobre el no volvemos a tener noticias que aludan a él con total seguridad. Tampoco sabemos cuándo falleció. En 1744 consta la existencia de un vecino de Vilanova de Lourenzá llamado José Martínez "Celis", a quien el Ayuntamiento de Mondoñedo tenía por "el mejor maesttro de fabrica de puentes y otras ôbras de arquitetura de piedra que ay en esta probinzia" ${ }^{52}$. Lo cierto es que en la abundante documentación manejada siempre lo hemos visto firmando como Celiz o Zeliz. Independientemente de su rúbrica, los manuscritos casi siempre lo citan como tal, y rara vez como Celis. De ahí que dudemos de si esta referencia alude a él, o, por el contrario, al maestro de obras homónimo que entre 1748-1766 trabajó en A Coruña y alrededores. Sea como fuere, las noticias que se tienen sobre este último hacen probable que se trate de un hijo suyo, pues según el Catastro de Ensenada (1752), dijo ser vecino de la ciudad herculina y contar con 49 años ${ }^{53}$. De ser cierto, habría nacido hacia 1703, cuando Martínez Celiz (padre) sumaba 23 años aproximadamente. Dicho Martínez Celis (hijo) pujó en 1748 por las obras del edificio de la Real Audiencia de Galicia ${ }^{54}$. A mediados de la década de 1750 realizó peritajes para el señor don Diego de $0 \mathrm{ca}^{55}$. En los últimos años del decenio trabajó junto con Alberto Ruibal en la fábrica de la iglesia coruñesa de San Nicolás ${ }^{56}$. En 1764 intentó adjudicarse las obras del Archivo General del Reino ${ }^{57}$. Dos años

templo financiado por el obispo Sarmiento véase GÓMEZ DARRIBA, Javier, La ciudad de Mondoñedo, opus cit., pp. 330-339.

52 Por aquel entonces el Concejo le encargó hacer un reconocimiento pericial del puente de Viloalle, perteneciente al Camino Real que atravesaba el río Masma a su paso por la feligresía mindoniense de Santa María de Viloalle. Celis tasó la reparación en 4.500 reales de vellón, pero no se ejecutó, GÓMEZ DARRIBA, Javier, "De piedra, arcos y agua. La construcción de puentes en el noreste de Galicia durante la Edad Moderna”, en Santander. Estudios de Patrimonio, 3, Santander, 2020, p. 259.

53 BARREIRO MALLÓN, Baudilio, La Coruña 1752 Según las Respuestas Generales del Catastro de Ensenada, Tabapress, Madrid, 1990, p. 150.

54 GARCÍA-ALCAÑIZ YUSTE, Julia, Arquitectura del Neoclásico en Galicia, Fundación Pedro Barrié de la Maza, La Coruña, 1989, p. 63.

55 SÁNCHEZ GARCÍA, Jesús Ángel, Mariñán. Pazo de los sentidos, Diputación de A Coruña, A Coruña, 1999, pp. 39, 47-48.

56 FOLGAR DE LA CALLE, María del Carmen, "La iglesia de San Nicolás de A Coruña”, en Brigantium, 5, A Coruña, 1984-1985, p. 160.

57 Arquivo Municipal de Mondoñedo, Carp. 951, Libro de Actas (1761-1770), 1764, fol. 50r. 
más tarde empedró la Calle de San Andrés ${ }^{58}$. También trabajó en las obras de mejora de las fortificaciones de la ciudad ${ }^{59}$. En definitiva, este personaje seguramente fue un hijo o pariente del Martínez Celiz aquí estudiado.

\section{Conclusiones}

Todo lo dicho hasta ahora sobre José Martínez Celiz nos permite afırmar que se trataba de un artista asturiano que conocía a la perfección la arquitectura que se practicaba en el Principado desde la segunda mitad del siglo XVII hasta las dos primeras décadas del XVIII, y muy especialmente la delineada por los arquitectos Francisco Menéndez Camina (padre e hijo). En el noreste de Galicia encontró un sitio donde establecerse y vivir cómodamente de su profesión. No se erigió en una figura de primer orden en el panorama arquitectónico de la región, al menos si lo comparamos con sus coetáneos Simón Rodríguez o Fernando de Casas. Pero sí constituyó uno de los maestros más importan- tes del obispado de Mondoñedo por dos razones fundamentales: porque introdujo en Galicia una serie de formulismos estructurales, articuladores y ornamentales nunca antes vistos en dicho Reino; y porque desarrolló una prolífica actividad artística en la citada diócesis, y muy especialmente en su capital. Allí se encontró con una variopinta clientela representada por el obispo, el Cabildo, las órdenes regulares, el Ayuntamiento o la hidalguía, que lo reclamaron como tracista, aparejador y perito de múltiples fábricas arquitectónicas. De hecho, durante el episcopado de fray Juan Muñoz y Salcedo (1705-1728) se convirtió junto con el lego cisterciense fray Agustín de Otero en el arquitecto más notable de la ciudad, y, en definitiva, en una de las personalidades que más contribuyó a la modernización de su arquitectura monumental. Por último, cabe destacar que, pese a resultar innovador en la Galicia de su tiempo, sus contribuciones y particularidades estilísticas no tuvieron continuidad en la región, ni tan siquiera en la provincia mindoniense en la que tantos años trabajó.

58 VIGO TRASANCOS, Alfredo, A Coruña y el Siglo de las Luces. La construcción de una Ciudad de Comercio (1700-1808), Universidade de Santiago de Compostela, Santiago de Compostela, Universidade da Coruña, A Coruña, 2007, pp. 144, 182.

59 SÁNCHEZ GARCÍA, Jesús Ángel, "Maestros de obras y aparejadores en la época contemporánea”, en El aparejador y su profesión en Galicia. De los maestros de obras a los arquitectos técnicos e ingenieros de edificación, Consello Galego de Colexios de Aparelladores, Arquitectos Técnicos e Enxeñeiros de Edificación, Santiago de Compostela, 2009 (ed. orig., 2001), p. 178. 